\title{
Gleichlauf von Verpflanzung und Kreuzung bei Froschlurchen.
}

\section{Schlusse auf die Zusammenfügung der Plasmabausteine.}

\author{
Von \\ Dr. Walther Schultz, \\ Arzt in Grandenz, Westpr. \\ Mit Tafel XII. \\ Eingegangen an 7. Oktober 1916.
}

Das Folgende ist eine kleine, leicht zu wiederholende Versuchsreibe zu meiner "Parallele von Verpflanzung und Kreuzung \&1).

Durch Vergleich unter möglichst gleichartigen Nebenbedingungen der Verpflanzung fand ich in früheren Arbeiten ein durchschnittliches Gleichlaufen von Verpflanzung und Kreazung bei Finken, Tauben, Htihnern und Hasenartigen.

Dies fuhbre ich darauf zurïck, daß beide Erscheinungsreihen von der Zusammensetzung des Lebensgrundstoffes, wohl der Chemie des Plasmas, abhängig seien und daß diese in Keim und Körper weitgehend die gleiche sei, weitgehend gleiche Arteigenheit bewahre. Ich kann mit besseren Grinden als vor meinen Versachen behaupten, daß die Kreuzung und uberhaupt jede Keimvereinigung eine Art Verpflanzung sei, und daß man aus den chemischen Verhältnissen der Verpflanzung Schlüsse anf die Verhältnisse der Keimanteile bei und nach ihrer Vereinigung machen diurfe.

Das Wiedererscheinen dem Auge entschwundener Erbanlagen bei mendelnder Vererbung und die Überlieferung von Geschlechtseigenschaften des anderen Geschlechtes, die Ursachen der Bastardunfruchtbarkeit, die Bedingungen der Bausteinaufnahme des lebenden Stoffes, insbesondere die Keimbansteinaufnahme (blastogene Insertion Rouxs), werden durch die Ergebnisse dieser Versuche etwas klarer.

1) Dieses Archiv Band 36, 37 (1913) und 41 (1915). 
Es ergab sich, daß die besonderen Nebenbedingungen, welche für Verpflanzungs- und Kreuzungserfolg, abgesehen von dieser Ähnlichkeit der Lebensgrundstoffe, in Betracht kommen, möglichst gleiche sein mlissen, wenn der Gleichlauf beider Erscheinungsreihen sich zeigen soll. Es ergab sich, daß die Ungleichbeit dieser Nebenbedingangen den Gleichlanf nur zu einem durchschnittlichen und lückenhaften macht und daß diese Nebenbedingungen auch oft die Versuchsreihen einfach abschneiden. Man sollte denken, daß diese Dinge schon längst erforscht seien, doch scheint das nicht der Fall zu sein. D. von Hansemann berichtet in einer Bemerkung über die Beziehungen der Bastardierung zur Transplantation $* 1$ ), wie weit er meine Ergebnisse vorausgenommen habe auf Grund ron Beobachtungen über leichte Hautverpflanzbarkeit zwischen Hase und Kaninchen, Haushand und Schakal. Der Grundgedanke scheint mir bei ihm derselbe zu sein wie in meinen Versuchen, seine Beweise scheint er aber noch nicht genau veröffentlicht zu haben. Wenn keine Angaben gemacht wurden, welche Verpflanzungsmethode er anwendet, wie lange die Verpflanzungen sich bielten, in welchen Punkten die Verpflanzungsdauer verglichen wurde, und überhaupt, ob unter gleichen Bedingungen die Ergebnisse verglichen wurden, so waren die Angaben noch zu allgemein gehalten.

In der Kenntnis der besonderen Bedingungen des Verpflanzungserfolges sind wir im einzelnen noch sehr zurïck. So kann ich nur vermuten, welche Gründe bei meinen Verpflanzungen zwischen kreuzbaren Enten- und Mausartigen die Darstellung des Gleichlaufs verhinderten. Ich vermute jetzt, daß der Fettreichtam der Entenhant vielleicht durch Fettumhüllung die Durchtränkung von Hautverpflanzungen in dem Unterhautzellgewebe so erschwerte, daß selbst arteigene Verpflanzungen nicht angingen. Auch vermute ich, daß der Albinismus meiner Mansartigen vielleicht die Hautstïckchen und überhaupt die Eiweißstoffe dieser Tiere wenig widerstandsfähig mache.

Eine unvermutete Übereinstimmung der Verpflanzungs- und Präzipitinreaktion zwischen Maus und Ratte ist mir erst jetzt bekannt geworden. Ich fand $\mathrm{ja}$, daß Hautverpflanzungen in ein Unterhantzellgewebe zwischen Maus und Ratte schnell zugrunde gehen, so daB. sich keine Verwandtschaft beider verrät. Nun lese ich in dem neuesten *Brehms Tierleben «, Band Nagetiere ${ }^{2}$ ), daß nach einem

1) Dieses Archiv Band 42, S. 126-127.

2) Verf. steht zurzeit im Felde. Es ist ihm daher nicht möglich, die Literaturangaben mit der üblichen Genauigkeit zu machen. 
Privatbrief P. Uhlenhuths an den Bearbeiter des *Brehm* Rattenund Mäuseblut sich mittels der Präzipitinreaktion ohne weiteres unterscheiden ließ, ja nicht einmal eine Verwandtschaftsreaktion zu erzielen war, wie z. B. zwischen Rind und Schaf. Iwanofrs Mischling zwischen Ratte und Mans wird daher auch vom Bearbeiter des •Brehm\& angezweifelt und es wird die Frage offen gelassen, ob nicht zwischen Ratte und Maus eine Verähnlichung durchaus fremder Stammformen bestehe.

Wie dies auch enden mag, sehr bemerkenswert ist doch die Übereinstimmung meines Verpflanzungsergebnisses mit der Präzipitinreaktion, deren Ergebnis ich bei Anstellung und Veröffentlichung jener Versuche durchans nicht kannte.

\section{Ausführung.}

Bei diesen Versuchen vergleiche ich oberfächliche sogenannte freie Hautverpflanzungen einfach mit bloßem Ange in bezug auf ihr Verwachsen und Erbaltenbleiben und ihre Häutungen als Wachstumserscheinungen and vermeide jede Berührung der Tiere mit antiseptischen Mitteln. Es ist in gewisser Weise vorteilhaft, daß wir hier Verpflanzungen vergleichen, die mit keinem Antiseptikum in Berihrung kamen. Ferner ist es vorteilhaft, daß wir bei den neuen Versuchen die verschiedenen Arteigenheitsanteile der Verpflanzung und ihre Erhaltung besser nnterscheiden and im Auge behalten als bei früheren Versuchsreihen. Auch scheint es mir ein gewisser Vorteil, daB bei dieser Versuchsreihe kein Hanstier benutzt zu werden braucht.

Quadratische Hautstiicke von 1 qcm wurden aus der Haut geschnitten und durch je eine Seidennaht an den Ecken und in der Mitte jeder Seite an die Ränder eines gleichartigen Loches in der Haut des Empfängers passend genäht. Es wurden nur die Hände mit Wasser und Seife gewaschen und die Instrumente mit Ather gereinigt. Die Tiere mit der frischen Pfropfang kamen sogleich in Gläser mit gewöhnlichem Brunnenwasser und Steinen, die ihnen gestatteten, nach Belieben das Wasser za verlassen. Sie saßen meist zu dreien in einem Einmacheglas von 4 Liter Inhalt. Solange es Fliegen gab, erhielten sie diese täglich, später nichts mehr, nur fast täglich frisches Wasser. Sie blieben auch im Winter in denselben Gläsern vor den Fenstern stehen. Hauptsache war, daß die Behandlung für alle gleichmäBig war. Ihr Gesundheitszustand war gut.

Wo nichts Besonderes bemerkt ist, sind die Verpflanzungen mit Rtickenhant und am Ruicken in regelrechter Lage ausgefubrt. Die 
Längenmaße messen von Schnauzenspitze bis After. Zur Artbestimmung benutzte ich , Fauna von Deutschland von BroHmer, 1914*. Die Kreuzungsergebnisse sind angefuhrt nach der Zusammenstellung von Przibram, "Experimental-Zoologie, 3«.

\section{Vergleich körpereigener und arteigener Verpflanzungen.}

Wasserfrosch. Rana esculenta L.

Alle Tiere etwa $7 \mathrm{~cm}$ lang.

Körpereigen.

Versuch 1 am 5. VIII. 15, vollkommen erhalten mit Drüsen, bis 1. II. 16 beobachtet.

Versuch 2 am 19. IX. 15 , um $90^{\circ}$ gedreht, vollkommen erhalten mit Drüsen, bis 1. II. 16 beobachtet.

Versueh 3 und 4 am 19. IX. 15. Austausch von Riucken- und Bauchhaut, vollkommen erhalten, Rückenhaut entwickelt Drüsen auch anf dem Bauche, bis 1. II. 16 beobachtet.

Die Versuche 1 bis 4 zeigen am 1. II. 16 noch ungeschrumpfte, Drüsenknötchen zeigende Haut, die am Farbwechsel des Wirtes teilnimmt.

Arteigen.

Versuch 5 and 6 am 15. VIII. 15, etwa 50 Tage erbalten, jedoch ohne Drisen zu zeigen.

Versuch 7 und 8 am 24. VIII. 15, verschiedene Färbungsspielarten, etwa 60 Tage erhalten, jedoch ohne Drïsen zu zeigen.

Die Versuche 5 bis 8 zeigen am 1. II. 16 keine sicheren Reste der Verpflanzung mehr.

Grasfroseh. Rana temporaria L.

Alle Tiere $6-7 \mathrm{~cm}$ lang.

Körpereigen.

Versuch 9 am 6. X. 15, um $180^{\circ}$ gedreht, vollkommen erhalten mit Drisen, nimmt am Farbwechsel des Trägers teil, bis 1. II. 16 beobachtet.

Arteigen.

Versuch 10 und 11 am 6 . X. 15, verschiedene Färbungsspielarten, etwa 100 Tage gut erhalten, jedoch keine Drüsenknötehen zeigend, dann dunkelt die Farbe und die Zeichnung wird verwaschen; am 1. II. 16 noch leidlich erhalten, etwas geschrumpft. 
Diese 11 gat vergleichbaren Versuche zeigen, daß die körpereigenen freien Hautverpflanzugen sich auch bei Lurchen wesentlich besser halten als die arteigenen, insbesondere beim Wasserfrosch.

Die auf dem eigenen Körper verpflanzten Hautstiucke haben mir bis zum Schlusse der Beobachtung, also 4 bis 6 Monate, eine ganz vollkommene Erhaltung gezeigt, keine körperfremde tat das. Insbesondere fiel mir auf, daß die Drüsen, welche als kleine Knötchen auf der Froschhaut sich erboben, bei allen körpereigenen Verpflanzungen sich wieder zeigten, nachdem sie in den ersten Wochen verschwunden waren, selbst bei Verpflanzung der drüsigen Ritckenhaut des Wasserfrosches auf seine glatte Bauchhaut.

Bei den körperfremden Verpflanzungen kamen diese Drüsenknötchen nie wieder.

Die Färbung der körpereigenen veränderte sich auf die Dauer nicht wesentlich, die der körperfremden wurde allmählich doch stets dunkler und verschwommener.

Nur die körpereigenen nahmen später wieder an den Farbwechselvorgängen der Froschlurche in gleicher Weise wie der Gesamtkörper teil.

Schrumpfung and AbstoBung der körpereigenen Stticke trat nicht ein oder war doch kaum bemerkbar, bei den körperfremden waren sie so stark, daß die vier vom Wasserfrosch ganz verloren gingen, und daß die beiden rom Grasfrosch auch so aussehen, als ob sie in einigen Monaten doch wohl ganz untergehen werden.

Wir treffen also die Unterschiede zwischen körper- und arteigenen Verpflanzungen (auto- und homoplastischer Transplantation), wie sie schon viele Forscher, zuletzt wohl am schönsten ScHöNE in seinem Buche "Die heteroplastische und homoioplastische Transplantation*, dargestellt haben.

Die freien Verpflanzungen der Daumenschwielen in der Froschhaut, welche Harms untersucht hat, sind nach meiner Deutung mit meinen Versuchen in guter Übereinstimmung. Auch schon aus meinen Versuchen, welche die langdauernde Eierstockverpflanzung auf Männchen 1900 und 1902 znerst lehrten, findet man, daß die Verpflanzung eine Einzelwesenkennzeichnung (Individualreaktion) ist.

Der fremde, ungewohnte Chemismus wird von den Verpflanzungen um so schwerer ertragen, je schwieriger die ubrigen Verpflanzungsbedingungen sind. Bei den vorliegenden Verpflanzungen maß auch eine mehr oder weniger dicke, schlecht durchblutete und 
schlecht durchtränkbare Led erhaut mit verpflanzt werden, besonders beim Wasserfrosch.

Ganz erheblich gebessert werden die Aussichten körperfremder Verpflanzung durch Blutsverwandtschaft, wie ich auch schon öfter zeigte. Fur die in dieser Arbeit berichteten kann Blutsverwandtschaft keine nennenswerte Bedeutung haben, weil Tiere zusammengebracht wurden, die an verschiedenen Orten wild gefangen wurden.

Die Ergebnisse arteigener Verpflanzung sind nun der Maßstab für die folgenden, insbesondere für die Verpllanzungen fremder, aber kreuzbarer Art.

\section{Die kreuzbaren Verpflanzungen.}

Die Verpflanzungen von kreuzbaren Arten nenne ich einfach "kreuzbar *, die von jenseits der Kreuzbarkeitsgrenze stehenden nenne ich $>$ nichtkreuzbar $\approx$.

Austausch zwischen Grasfroseh - Rana temporaria L. und Moorfrosch - Rana arvalis Nilson.

Versuch 12 und 13 am 15. X. 15, Länge je $6 \mathrm{~cm}$, siehe Fig. 1 und 2 (Taf. XII).

Vers. 12. Die Haut von Moorfrosch auf Grasfrosch glatt eingeheilt, gut erhalten bis zum vorläufigen Abschluß der Beobachtung 1. II. 16, also 108 Tage. Drtisen zeigen sich nicht. Zeichnung und Farbe aber bleiben bis zuletzt fast unverändert and auch die Schrumpfung ist bis zuletzt kaum bemerkbar.

Vers. 13. Die Hant von Grasfrosch auf Moorfrosch ebenfalls glatt eingeheilt, gut erhalten bis 1. I. 16, also fast 80 Tage. Drusen zeigen sich nicht. Zeichnung und Farbe aber bleiben gut erbalten. Geringe Schrumpfung. Etwa am 1. I. 16 beginnt aber die Verpflanzung sich abzustoßen, vielleicht infolge äußerer Reibung. Sie hinterläßt eine lochartige Wunde.

Versuch 14 und 15 am 18. X. 15. Grasfroseh $6 \mathrm{~cm}$, Moorfrosch $4 \mathrm{~cm}$ lang.

Vers. 14. Die Haut von Moorfrosch auf Grasfrosch glatt eingeheilt. Farbe und Zeichnung bleiben etwa 90 Tage gut, dann werden sie dunkel und verschwommen. Drüsen zeigen sich nicht. Am 1. II. 16, also nach 105 Tagen, ist aber die Erhaltung noch immer leidlich.

Vers. 15. Die Haut vom Grasfrosch auf dem Moorfrosch geht nach 24 Tagen durch Abreißen bei der Besichtigung verloren. 


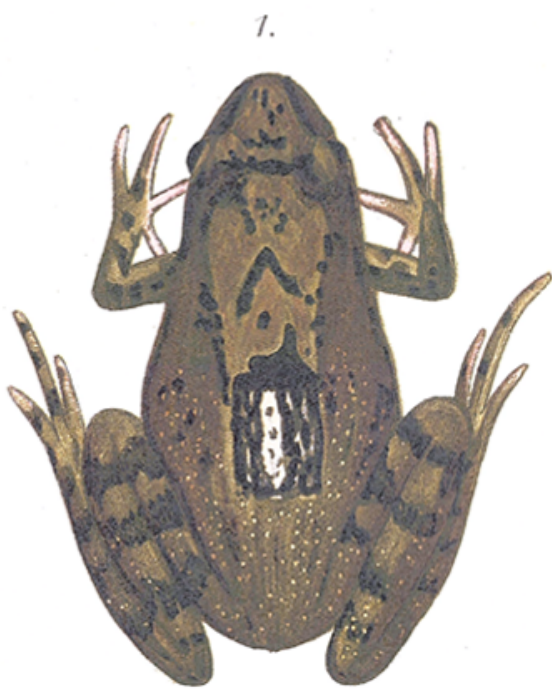

2.
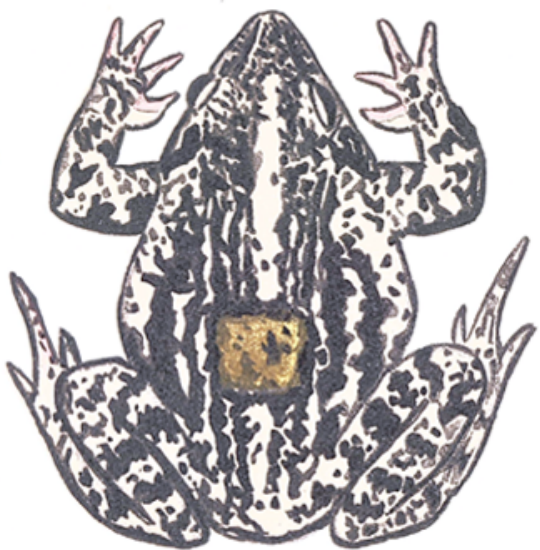

5.

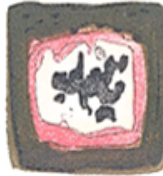

3.

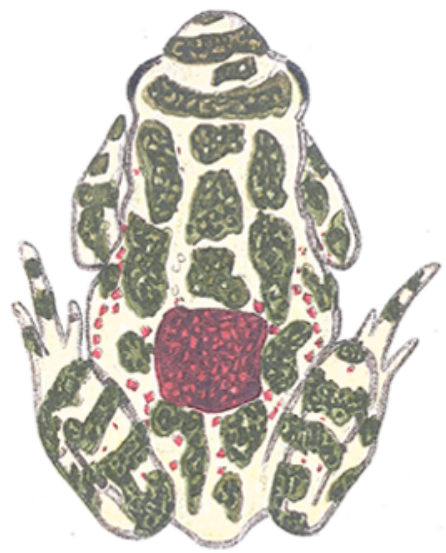

4.

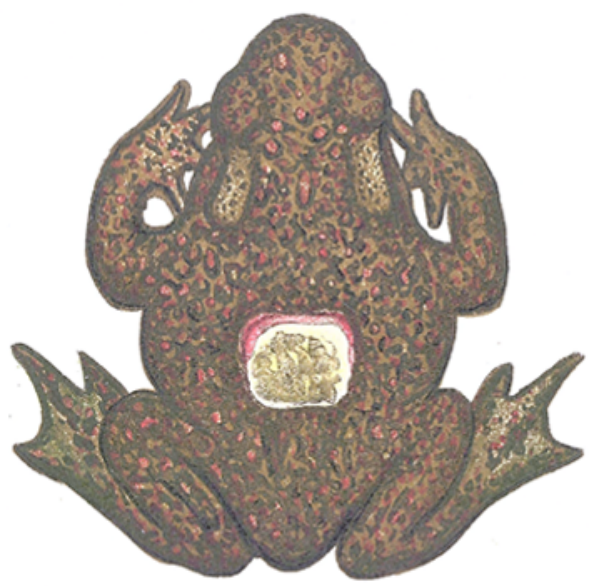


Versuch 16 und 17 am 18. X. 15. Grasfrosch $6 \mathrm{~cm}$, Moorfrosch $4 \mathrm{~cm}$ lang.

Vers. 16. Die Haut von Moorfrosch auf Grasfrosch verbält sich bis zum 105. Tage genau so gut wie die des Versuches 14.

Vers. 17. Die Haut vom Grasfrosch auf dem Moorfrosch zeigt nach einigen Tagen wunde, offene Umrandung, weil einige Fäden durchschnitten. Die Verpflanzung wächst trotzdem fest und stößt sich erst nach 70 Tagen allmählich ab, nachdem sie Farbe und Zeichnung so lange gut bewahrte, aber keine Drüsen zeigte.

Die Farbe aller dieser Grasfrösche war braungelb mit schwach grtinlichem Tone auf dem Rücken. Die Farbe der Moorfrösche war grau bis hellgran-braun mit schwarzer Zeichnung. Die Moorfrösche zeigten alle hellen Mittelrückenstreif, die Grasfrösche nicht. Diese Eigentümlichkeiten bewahrten die Verpflanzungen treu.

Die Erhaltung dieser Verpflanzungen war also so gut wie bei den arteigenen, die zum Vergleich dienen können. Soweit Moorfrosch auf Grasfrosch gepflanzt war, wurden drei Fälle mit Leichtigkeit über den 100. Tag erbalten, im amgekehrten Verhältnis hielten sich die Verpflanzungen anch fast bis zum 80 . Tage gut.

Austausch zwischen Erdkröte - Bufo vulgaris Laur. und Wechselkröte - Bufo viridis Laur.

Versuch 18 und 19 am 21. X. 15, je $6 \mathrm{~cm}$ lang (Fig. 3 Taf. XII).

Vers. 18. Erdkröte mit Wechselkröteneinpflanzung zeigt zunächst gute Einheilung und bis zum 30. Tage auch gute Erhaltung, dann allmählich schmutzige Verfärbung und Verfall.

Vers. 19. Die Erdkrötenhaut auf der Wechselkröte heilt gut ein und bleibt tadellos erhalten bis zum vorläufigen Beobachtungsabschluß 1. II. 16, also rund 100 Tage. Die Verpflanznng ließ bis in die letzte Zeit hinein wiederholt Häutungsschichten abziehen. Sie hatte die braunrote Farbe mit roten Druisenwarzen bewahrt, wie sie beim Verpflanzungseingriff selbst war, während das Tier, von welchem sie stammte, inzwischen sich mehr schmutzig braungrau umgefärbt hatte. Jenseits des 100. Tages wurde auch die Verpflanzung so schmutzig braungrau mit rötlichen Warzen, wie es die regelrechte Haut der Erdkröte von Fig. 4 (Täf. XII) zeigt.

Die Warzen der Verpflanzung waren etwas verkleinert, aber erbalten.

Versuch 20 und 21 am 24. X. 15, je $3 \mathrm{~cm}$ lang.

Vers. 20. Die Erdkröte mit Wechselkrötenhaut stirbt nach 15 Tagen. Das Pfropfstuick zeigt sich noch nicht fest verwachsen, nur fest verklebt. 
Vers. 21. Die Erdkrötenhant anf der Wechselkröte zeigt sich anfangs mit wunden Stellen umgeben. Nach einigen Wochen aber überheilen diese Wundflächen ganz, so daß die Verpflanzung jetzt tadellos eingewachsen war. Sie verhielt sich sonst wie Vers. 19. Es ließ sich noch nach iuber 100 Tagen eine Häntungsschicht abziehen and sie erhielt ihre ursprüngliche Farbe gut, auch ihre Warzen verkleinerten sich, blieben aber erhalten.

Versuch $22 \mathrm{am} 21$. X. 15. Alte Erdkröte von etwa $8 \mathrm{~cm}$ Länge erhielt am Rücken die Rückenhaut einer jungen, etwa $21 / 2 \mathrm{~cm}$ langen Wechselkröte. Die Verpflanzung verklebt gut, bleibt aber von Wunden umgeben, wird nach 20 Tagen dunkel, um mit 30 Tagen allmählich zu zerfallen.

Auch diese kreuzbaren Verpflanzungen zeigen also zwei Fälle, die bis tiber den 100. Tag noch tadellos erhalten waren und noch Häntungsschichten abziehen ließen. Die schlechtesten Hautverpflanzungen zwischen diesen kreuzbaren Krötenarten zerfielen doch erst vom 30. Tage ab.

Meine kreuzbaren Frosch- und Krötenverpflanzungen waren den arteigenen Vergleichsverpflanzungen so ähnlich, daß wir auch für die Keimverbindungen der kreuzbaren etwa gleiche Bedingungen annehmen müssen wie für die arteigenen, das heißt gemeinsame Lebensfähigkeit nach der Verschmelzung, wenn der Gegensatz zu den nichtkreuzbaren in weiteren Versuchen sich ebenso scharf ausprägt wie in den Versuchen dieser Arbeit.

\section{Die nichtkreuzbaren Verpflanzungen außerhalb der Kreuzbarkeits- grenzen.}

Zum Vergleich mit den kreuzbaren Verpflanzungen dienen am besten die folgenden, zwischen Fröschen einerseits und Kröten andererseits, ferner die zwischen Fröschen und Kröten einerseits und Schwanzlurchen andererseits. Zwischen diesen Gruppen waren ja die Befruchtungsergebnisse meist nur unregelmäBige Furchungen. Das günstigste Ergebnis zwischen diesen Gruppen war eine ausgeschlüpfte Larve bei der Kreuzung Bufo viridis $\sigma^{T} \times$ Rana esculenta 우.

Ich habe eine genügende Zahl Vergleichsversuche dieser Nichtkrenzbarkeit von denselben vier Arten, mit denen ich die kreuzbaren Verpflanzungen machte. 
Versuch 23 und 24 am 31. X. 15. Grasfrosch und Erdkröte. Länge je $6 \mathrm{~cm}$. Austausch.

Vers. 23. Die Froschhaut auf der Kröte nach 17 Tagen schon schmutzig grau, ohne Zeichnung. Vom 20. Tage schneller Zerfall.

Vers. 24. Die Krötenhant anf dem Frosch wird erst schwärzlichgrau und ist am 28. Tage auch schon so zerfallen und abgestoßen, daß nar einige weiße Bindegewebsschichten noch auf dem Froschriicken kleben.

Versuch 25 und 26 am 5. XI. 15. Grasfrosch und Erdkröte je $3 \mathrm{~cm}$ lang. Austausch.

Kröte stirbt nach 10, Frosch nach 13 Tagen, die Verpflanzungen nur locker verklebt.

Versuch 27 und 28 am 1. X. 15. Grastrosch und Wechselkröte je $6 \mathrm{~cm}$ lang. Austausch.

Kröte stirbt nach 13, Frosch nach 10 Tagen. Froschhaut schon in Ablösung, Krötenhaut nur verklebt.

Versuch 29 und 30 am 30. X. 15. Moorfrosch and Wechselkröte je $6 \mathrm{~cm}$ lang. Austausch.

Beide Verpflanzungen nach 20 Tagen in vollem Zerfall.

Versuch 31 und 32 am 7. IX. 15. Erdkröte $6 \mathrm{~cm}$ und Wasserfrosch $7 \mathrm{~cm}$ lang. Austausch.

Vers. 31. Die Froschhaut auf der Kröte löst sich schon nach 16 Tagen in den oberen, Farbzellen tragenden Schichten zu einer grauen Schmiere.

Vers. 32. Die Krötenhant auf dem Frosche ist schon nach $16 \mathrm{Ta}-$ gen so zerfallen, daß nur eine weißgraue Unterschicht bleibt.

Versuch 33 am 2. XI. 15. Von junger Wechselkröte, $2 \frac{1}{2} \mathrm{~cm}$ lang, auf alten Wasserfroseh, $7 \mathrm{~cm}$ lang.

Am 20. Tage in vollem Zerfall.

Versuch 34 am 4. XI. 15. Grasfrosch, $6 \mathrm{~cm}$ lang, erhält von Teichmolch, Molge vulgaris Laur., ein Stück der gelbroten Bauchseite auf den Riticken.

Die Verpflanzung zerfält and löst sich etwa am 10. Tage, nachdem sie vorher hell und verwaschen geworden war.

Versuch 35 am 22. X. 15. Moorfrosch, $6 \mathrm{~cm}$ lang, erhält von Feuersalamander Bauchhaut auf den Rücken.

Moorfrosch stirbt am nächsten Tage, obwohl er bisher ganz munter war. 
Versuch 36 am 22. X. 15. Erdkröte, 6 cm lang, erhält von Feuersalamander Bauchbaut auf den Ricken.

Vom 8. Tage ab wurden die schwarzen Flecken in der rotgelben Verpflanzung grau und am 13. Tage ist die Verpflanzung in Zerfall und Ablösung.

Meinen 11 kreuzbaren Verpflanzungen kann ich also 14 vollgültige nichtkreuzbare Vergleichsverpflanzungen entgegenstellen.

Die längste Erhaltungsdauer war bei den 11 kreuzbaren in 5 Fällen uber 100 Tage, bei den 14 nichtkreuzbaren in 1 Fall etwa 25 Tage.

Frubbzeitig starben von den kreuzbaren 1 , von den nichtkreuzbaren 5 .

Die durchschnittliche Erhaltungsdauer der länger lebenden kreuzbaren war 75 Tage, die der nichtkreuzbaren 18 Tage.

Worin ich auch die kreuzbaren und nichtkreuzbaren vergleiche, Tod, Ablösung, Zerfall, Schrumpfung, Verschwimmen der Zeichnung, alles tritt bei den nichtkreuzbaren viel fruher auf als bei den krenzbaren, und lange andauernde Häutungen fand ich nur bei Verpflanzungen der letzteren Art.

So muß man also vermuten, daß durch zu große Verschiedenheiten des lebenden Grundstoffes ein längeres Zusammenleben dieser Grundstoffe ebensowenig bei Kreuzung wie bei Verpflanzung für diese nichtkreuzbaren Arten möglich ist.

Der Laubfrosch, Hyla arborea Laur., steht den Rana-Arten anscheinend näher als diese den Bufo-Arten, Kreuzungsergebnisse von Rana und Hyla sind mir nicht bekannt, vielleicht steht er auch außerhalb der Grenzen einer Kreuzbarkeit, welche bis zur Verwandlung gelangende Nachkommen liefert. Die Verpflanzungsergebnisse zwischen meinen Rana-Arten und dem Laubfrosch waren jedenfalls schlechte und ich glaube, daß durch diese Ergebnisse die Zahl der schlechte Ergebnisse liefernden nichtkreuzbaren Verpflanzungen noch vermehrt wird. Ich glaube aber auch, daß stärkere Ungleichheit der Nebenbedingungen hier eine Rolle spielt.

Versuch 38 und 39 am 21. IX. 15. Grasfrosch und Laubfrosch je $4 \mathrm{~cm}$ lang. Austausch.

Grasfrosch stirbt nach 7 Tagen, Laubfrosch nach 8 Tagen. Laubfroschhaut in Ablösung. Grasfroschhaut sitzt noch fest, aber nur verklebt. 
Versuch 40 und 41 am 3. XI. 15. Moorfrosch und Laubfrosch je $4 \mathrm{~cm}$ lang. Austausch.

Moorfrosch stirbt mit 5 Tagen, Laubfrosch mit 8 Tagen, Laubfroschhant an den Rändern blutig schmutzig, Moorfroschhaut nicht abgelöst, aber nur durch Verklebung haftend.

\section{Verpflanzung zwischen nichtkreuzbaren, aber innerhalb der Kreuz- barkeitsgrenzen stehenden Arten.}

Dies Verhältnis besteht zwischen Wasserfrosch und Grasfrosch. Kreuzungen zwischen diesen Arten kamen über die Aufänge der Entwicklung nicht hinaus. Aber Tiere, welche in der systematischen Stellung weiter auseinander stehen, nämlich Rana fusca $O^{\top} \times$ Pelobates fuscus $Q$ brachten es noch bis zu verwandelten Nachkommen.

Zwischen Wasserfrosch und Grasfrosch erhielt ich nun ein sehr gutes Verpflanzungsergebnis.

Versuch 42 und 43 am 20. IX. 15. Wasserfrosch $7 \mathrm{~cm}$, Grasfrosch $7 \mathrm{~cm}$. Austausch.

Vers. 42. Die Wasserfroschhaut auf dem Grasfrosch beginnt nach 40 Tagen teilweise zu zerfallen, aber erst nach 60 Tagen sind die letzten Reste verschwanden, die noch fast bis zuletzt ihre grüne Farbe bewahrten.

Vers. 43. Die Grasfroschbaut anf dem Wasserfrosch hielt sich 90 Tage fast unverändert in Größe und Zeichnung, tadellos eingeheilt, nur ohne Drlisenknötchen. Dann schrumpfte sie langsam, dunkelte und warde in der Zeichnung undentlicher. Aber noch am Schluß der Beobachtung, also nach mehr als 130 Tagen, war die Verpflanzung in der halben ursprïnglichen Größe erbalten und zeigte noch ziemlich dentlich ihre urspringliche Farbe und Zeichnung.

Versuch 44 am 2. XI. 16. Moorfrosch erhielt Wasserfroschhant. Moorfrosch stirbt am 17. XI. Verpflanzung blutig, schmutzig graugritn.

Das gute Verpflanznngsergebnis zwischen Wasserfrosch und Grasfrosch ist übrigens auch von HARMS und anderen erhalten worden.

Ich schließe, daB ebenso wie die Systematik auch die Verpflanzungsforschung uns lehrt, daß die lebenden Stoffe von Grasfrosch und Wasserfrosch an sich wohl ein Zusammenleben gestatten, und daß wohl nur besondere Nebenumstände der Kreuzung, sei es Reifezeit oder sonst etwas, das Kreuzungsergebnis schädigten.

Wir können erkennen, auf Grund der Verpflanzungsergebnisse, wo in der Natur die Nichtkreazbarkeit nicht 
durch den Bau des Plasmas, sondern durch sNebenumständec bewirkt wird.

Freilich aus den vorliegenden Versuchen darf man es nur schließen unter der Voraussetzung, daß der Gegensatz zu den nichtkreuzbaren Verpflanzungen sich immer wieder bestätigen wird.

\section{System und Kreuzung bei den Wasserfroschverpflanzungen.}

Wir sahen schon, daß beim Wasserfrosch Hautverpflanzung auf gleichem Tiere sich bei weitem am besten hielt. Zwischen verschiedenen Tieren der gleichen Art hielt sie sich 50 bis 60 Tage. Auf Grasfrosch hielt sich die Wasserfroschhant 40 Tage gut. Die Grasfroschhaut auf dem Wasserfrosch aber hielt sich sogar bis über den 130. Tag noch leidlich. Artfremde Verpflanzung war also erfolgreicher als arteigene.

Schon auf diesem kurzen Wege zeigt sich ein Abweichen der Verpflanzang vom Gleichlauf mit dem System. Den Grund werden wir später nennen.

Weitere Versuche mit dem Wasserfrosch ergeben auch, daß zwar durchschnittlich ein Gleichlauf von Verpflanzung und System bestebt, daß aber im einzelnen Abweichungen von der strengen Parallele vorkommen.

Versuch 45 und 46 am 20. X. 15. Wasserfrosch $7 \mathrm{~cm}$, Laabfrosch $3 \mathrm{~cm}$ lang. Austausch.

Laubfrosch stirbt nach 12 Tagen. Wasserfroschhaut nur verklebt. Mitte noch gut gefärbt. Ränder blutig schmutzig zerfallend.

Laubfroschbaut auf dem Wasserfrosch mit 14 Tagen sehr mißfarbig, löst sich mit 18 Tagen ab.

Versuch 31 und 32 berichtete schon, daß Verpflanzungen zwischen Wasserfrosch und Erdkröte in beiden Verhältnissen mit 16 Tagen zerfielen.

Versuch 33 zeigte Verpflanzung von junger Wechselkröte anf Wasserfrosch, wie auch bereits berichtet, mit 20 Tagen in vollem Zerfall.

Versuch 47 und 48 am 21. IX. 15. Wasserfrosch $7 \mathrm{~cm}$, Feuersalamander ausgewachsen. Austausch.

Wasserfroschhaut auf Feuersalamander ist am 8. Tage ganz schwarz geworden. Ganz auffällig hat sich ihr urspruingliches Grín in Schwarz verwandelt. Am 12. Tage löst sich die Haut ab. Die Salamanderhaut auf dem Wasserfrosch löst sich mit 10 Tagen ab. 
Vergleichen wir wieder diese Verpflanzungen außerhalb der Kreuzbarkeitsgrenzen mit den innerhalb der Kreuzbarkeitsgrenzen gelegenen, wenn anch nicht tatsächlich gekreuzten zwischen Wasserfroseh und Grasfrosch, so wird uns wieder der Gleichlauf von Verpflanzung and Kreuzung offenbar.

Die Grtinde der Abweichangen vom Gleichlauf zwischen Verpflanzung und System liegen in denselben Nebenbedingungen, die ebenso oder sogar noch leichter auch den Gleichlauf von Verpflanzung und Kreuzung stören.

Die hier in Betracht kommenden Nebenbedingungen sind diejenigen, welche neben der chemischen oder sonstigen Arteigenheitszusammensetzung des Lebensgrundstoffes bestehen.

\section{Die Färbungserscheinungen an den Pfropfstïcken.}

Wenige Worte uber diese Beobachtungen:

ln der ersten Zeit nach der Verpflanzung zeigt sich fast allgemein eine Aufhellung der Verpflanzungen.

Teilnahme an dem Farbwechsel des Wirtskörpers der Froschlurche trat nur bei den körpereigenen Verpflanzungen auf, und zwar erst nach mehreren Wochen.

Das Farbmuster blieb in seiner Eigenart trotz der Verlagerung fast stets unverändert, solange nicht Zerfall und starke Schrampfung wirkten.

Bei den körpereigenen Wasserfroschverpflanzungen bemerkte ich, $\mathrm{daB}$ in Vers. 1 die helle Mittellinie etwas nach rechts verlagert war. Sie blieb es dauernd, in 6 Monaten formte sich nichts am. In Vers. 2, wo die belle Mittellinie quer gelagert war ond das ganze Sttick im Laufe der Zeit quer gereekt wurde, konnte ich die helle Mittellinie nach 4 Monaten nicht mehr deutlich erkennen.

Weiße Bauchhaut in dunkle Rückenhaut gepflanzt and umgekehrt dunkle Rückenhaut auf den weißen Bauch gepflanzt blieben in den Vers. 3 und 4 von dieser Verlagerung geradezu unbeeinflußt. Ganz wie es in diesem Archiv schon von Fredinand. WinkLer geschildert warde.

Die Spielarteigenschaften der Pflanzstícke in den Vers. 7 and 8 und 10 und 11 blieben trotz der allmählichen Verdunkelung und des sicheren oder anscheinenden Unterganges bis zuletzt erkennbar. In Vers. 7 und 8 war die eine Wasserfroschhaut mehr bläulichgrïn, die 
andere mehr gelbgrtin. In Vers. 10 und 11 war die eine Grasfroschhaut mehr rötlich punktiert, die andere mehr einfach braun marmoriert.

Die langsame Verdunkelung in späterer Zeit der arteigenen and kreuzbaren Verpflanzungen schilderte ich schon.

Auffallend war, daß die grtine Wasserfroschhaut auf einem anf der Oberseite ganz schwarzen Feuersalamander sich bald ganz schwarz färbte. Dies sah aus wie eine vom Wirtskörper bewirkte, ihm selbst gleichsinnige Veränderung der Eigenart des Pfropfstỉckes.

Übrigens waren ebenso wie dieser auch alle anderen Salamander, die ich im Herbst 1915 im Walde bei Tuchel, Westpr., fand, im ganzen 10 Stick, große und kleine, an der Oberseite ganz schwarz, ohne gelbe Flecken.

Zweifel am Leben der homoioplastischen treien Hautverpflanzungen nicht blutsverwandter Froschlurche. - Ein Vorbehalt der theoretischen Wertung.

Ich will -nicht verheimlichen, daß ich diese Überpflanzangen der Froschlurehhaut in allen Fällen mit Ausnahme derjenigen auf dem gleichen Einzelwesen nicht mit Sicherheit als echte Verpflanzungen ansehe, bei denen das Verpflanzungsstick wirklich am Leben bleibt.

Eine Anzahl Forscher geben Ergebnisse, die von den von mir berichteten nicht wesentlich verschieden sind, ohne Umstände in dem Sinne wieder, als ob die Verpflanzungen noch lebten. Gegen die Deutung der Versuche von Harms, KaMMERER und anderen tiber homoioplastische Lurchbautverpflanzungen in dem Sinne, als ob hier die Verpflanzungen längere Zeit gelebt hätten, hege ich Mißtrauen. Selbist die Möglichkeit, wiederholt Häutung®schichten abzuziehen, beweist nicht streng, wie lange die Verpflanzungen lebten.

Es kann sich deshalb möglicherweise nur um Vorgänge handeln, gleich als ob CARREL Hundeaorta in Menschenaorta erfolgreich einpflanzt. Es kann ein gemustert gefärbtes totes Bindegewebe, eine Art buntes Leder sein, das wir mit Erfolg einpflanzen, implantieren, nicht transplantieren.

Wenn aber der Gegensatz zwischen kreuzbaren und nichtkreuzbaren Verpflanzungen auch bei dieser Versuchsanordnung sich weiter bestätigt, muß man daraus doch annähernd gleiche Schltusse ziehen, wie beim Nachweis der tatsächlichen Lebensdaner der Verpflanzung.

An der Berührungsstelle der Einpflanzung mit dem Wirtsgewebe werden sich biochemische Arteigenheits-arteigene Schutzstoffreak tionen abspielen, die alle im wesentlichen gleiche Schlisse gestatten. Ich 
erinnere hier nur nochmals an die sehr bemerkenswerte Übereinstimmung zwischen Präzipitinreaktion und Verpflanzungsreaktion zwischen Ratte und Mans (Uhlenhuth, Walther Schultz, Iwanoff).

Soll es ein Zufall oder eine Täuschung sein, daß diese Versuche wieder mit den fast tansend anderen Versuchen, die ich unter besseren Prtifungsmaßnahmen auf Erhaltung des Lebens der Verpflanzungen machte, in so guter Übereinstimmang sind?

Ich wage es deshalb doch, auch dieser Versuchsreihe einige theoretische Betrachtungen hinzuzufügen, die mindestens in bezug auf meine vielen früheren sicheren Befunde tiber Gleichlauf von Verpflanzung und Kreuzung klärenden Wert haben.

\section{Die Nebenbedingungen.}

Zunächst sehen wir bei Verwendung 1 qem großer Verpflanzungen kleinere Tiere leichter sterben als größere, auch noch, wenn wir die Größe des Verpflanzungsstuckes etwas vermindern, so daß wir wenigstens keine erheblichen Blntgefäße beim Ausschneiden verletzen.

Man kann das auch noch aus folgenden körpereigenen Grasfroschverpflanzungen kleiner Tiere seben:

Versuch 49 am 19. IX. 15. Körpereigene Verpflanzungen auf $31 / 2 \mathrm{~cm}$ langem Grasfrosch. Tod des Tieres nach 10 Tagen.

Versuch 50 and 51 am 7. IX. 15. Grasfrosch, $4^{1 / 2} \mathrm{~cm}$ lang, körpereigener Austausch von Ruicken- and Bauchhaut. Tod nach 5 Tagen.

Nan gibt es Arten, die tiberhaupt klein bleiben, wie den Laubfrosch. Auch körpereigene Versuche ergeben wegen seiner Kleinheit leichten Tod des Tieres.

Versuch 52 am 2. X. 15. Laubfrosch, körpereigene Verpflanzung. Tod nach 6 Tagen.

Versuch 53 am 3. XI. 15. Desgl. nach 5 Tagen.

Die Nebenbedingungen, welche den Gleichlauf zwischen Verpflanzung und Kreuzung stören, können demnach auch durchaus regelrechte Arteigenheiten sein.

Wir müssen zar Darstellung des Wirkens der arteigenen Zusammensetzung des Plasmas Verhältnisse wählen, welche die Nebenbedingungen gleich machen oder rechnerisch die Nebenbedingungen anf gleiches Maß zurückführen lassen. 
Wenn wir die verschiedenen Tierarten, die wir vergleichen wollen, als ungerade Zahlen betrachten, so maß unsere Versuchsanordnung sie alle auf gleichen Nenner bringen.

Soweit wir Parallelen von Verpflanzung and Kreuzung schon tatsächlich fanden, haben wir offenbar auch die richtige Versuchsanordnung in diesem Sinne gefunden, z. B. bei der Verpflanzung kleiner Hautstückchen von Vögeln und Säugern in das Unterhantzellgewebe.

Bei genauer Kenntnis des Wertes der Nebenumstände können wir sie umrechnen, auch wenn sie verschieden sind.

Ich vermute, daß bei Froschlurchen auch die Dicke der Haut, die Temperatur, bei der die Verpflanzungen gemacht werden, das Gift der Hautdrüsen Nebenumstände sind.

Bei dem Reichtum der wechselnden Nebenumstände ist es richtig, für die Allgemeinheit der Verpflanzungen nur einen durchschnittlichen Gleichlauf von Verpflanzung and System, Kreuzang und System und $z$ wischen Krenzung und Verpflanzang zu erwarten, so wie ihn auch die Tatsachen ergeben.

Es scheint, daß bei den vorliegenden Versuchen der Gleichlauf nur noch besser erkennbar wird, wenn wir die Nebenumstände verrechnen.

Nehmen wir die Gesamtgröße: Weshalb gediehen kleinere Kröten mit fremdartiger Krötenhant besser als größere Frösche mit ebenderselben?

Nehmen wir die Lederhautdicke des Verpflanzungsstiickes: Weshalb gedieh viel dickere fremdartige Krötenhant auf Kröten besser als viel dünnere, leichter ernährbare Frosch- und Schwanzlurchhaut?

Nehmen wir die Absonderung giftiger Sekrete: Weshalb gedieh Krötenhaut auf andersartigen Kröten, die reichlich ein in gewissem Maße giftiges Sekret abgeben, besser als auf Fröschen, die gar nichts derartiges absonderten?

Gerade bei den Froschlurchen ergab die Kreuzung zwischen den gleichen Arten oft sehr verschiedene Erfolge, je nachdem, ob das gleiche Geschlecht von der einen oder anderen Art gewählt wurde. Ebenso sehen wir bei Grasfrosch-Wasserfrosch, Grasfrosch-Moorfrosch, Erdkröte-Wechselkröte verschiedene Erfolge, je nachdem welcher Paarteil Geber oder Empfänger der Verpflanzung ist. So ist anch das Geschlechtserzengnis zweier Spielarten oder Kreuzbaren gelegentlich verschieden bei wechselndem Geschlechtsverhältnis.

Bei Verpflanzung und Kreuzung verursachen verschiedene Nebenbedingungen der Paarlinge ein verschiedenes Überkrenzergebnis. 
Es sei hier am Schlusse der Versuche noch angeführt, wie sich erstens die Verpflanzungswunden und zweitens die Verpflanzungsstilcke verhalten, wenn keine Einpflanzung vorgenommen wird.

Versuch 54 am 15. VIII. 15. Wasserfroseh, $7 \mathrm{~cm}$ lang. Ausschnitt der Ruckenhaut quadratisch, $1 \mathrm{qcm}$ Wunde sich selbst uberlassen.

Die Wundränder verklebten zunächst mit der inneren Wand des Rückenlymphsackes. Der Ausschnitt wird dann von einer bindegewebigen grauen Haut überwachsen, die sich zwischen den Wundrändern hinspannt. Diese Haut hebt sich weiterhin von der Innenwand des Ruickenlymphsackes ab und stellt nun die ursprünglichen Verhältnisse des Rtickenlymphsackes wieder her, indem sie das Loch in seiner Außenwand verschließt. Sie schrumpft langsam zusammen und taberzieht sich anscheinend zuletzt mit schwarzer Farbstoffschicht, ohne da $B$ sichere Regenerationserscheinungen weiter bemerkbar werden.

Versuch 55. Ausgeschnittene Stlicke der äußeren Hant von Fröschen und Kröten hielten sich wochenlang im Wasser gut in der Farbe unter Abgabe von Häutungsschichten. Da sie sich zum Teile besser hielten als nach Verpflanzung auf nichtkreuzbare fremde Arten, deutet dies darauf hin, daß Lysine oder Abwehrfermente oder ähnliche Schutzkörper arteigener Natur auch bei der Zerstörung und Auflösung der Verpflanzungen tätig waren.

\section{Verpflanzen sich gleiche Anteile des lebenden Stoffes auch bei artfremder Herkunft leichter aufeinander?}

Die Verpflanzung Erdkröte auf Wechselkröte hielt sich nach unseren Versuchen wesentlich besser als die umgekehrte.

Es scheint nun, daß die Erdkrötenhaut mehr Anteile besitzt, die auch auf der Wechselkröte schon vorhanden sind, als umgekehrt.

Die Erdkrötenhaut bestand bei meinen Versuchstieren aus rötlich gefärbten Wärzchen, deren zwischenliegende Haut zeitweilig auch mehr grau erschien. Die rötlichen Wärzchen finden wir auf der Wechselkröte auch, die Fig. 4 (Taf. XII) zeigt sie angedeutet, und graue Anteile hat die Wechselkrötenhaut reichlich. Danach kommt also die Erdkrötenhaut auf der Wechselkröte auf einen Boden, der seine Fähigkeit, ihre Anteile zu tragen, bereits gezeigt hat.

Anders verhält es sich umgekehrt. Die Wechselkrötenhaut enthält ausgedehnte grine Anteile, die ich auf den von mir verwendeten Erdkröten nicht fand. Es gibt freilich auch griinliche Erdkröten. 
Dasselbe Verhältnis zeigte sich auch bei meinen GrasfroschMoorfroschverpflanzungen.

Die Moorfroschhant, welche ich verpflanzte, war hellgran-bräunlich und schwarz, also aus Anteilen zusammengesetzt, die auf dem Grasfrosch auf Seite und Bauch reichlich vorkommen. Dagegen zeigte die Grasfrosehhaut braungrinnliche Anteile, die auf meinen Moorfrösehen fehlten, wenn sie freilich auch auf anderen vorkommen.

Dementsprechend hielt sich meine Moorfroschhautverpflanzung besser.

Die grüne Wasserfroschhaut hielt sich, sobald sie körperfremd war, auf den grünen Wasserfröschen schlechter als die branne und graue Grasfroschhaut.

Wenn es kein Zufall ist, daß in allen drei Fällen die mehr gruinlichen Hautstïcke sich schlechter bielten, so könnte doch die grüne Farbe irgendeine besondere Eigenschaft, welche die Verpflanzung erschwert, z. B. Dicke, andeuten.

Es gibt offenbar auch Unähnlichkeiten, die eine Verpflanzang erleichtern, im Vergleiche zu Ähnlichkeiten, die sie erschweren. Es ist anch noch zu schwer, alle Einzelbedingungen der Verpflanzung und Krenzung za uberschauen. Immerhin bleibt wahr, daß bei den am besten haltbaren artfremden Verpflanzungen dieser Versuchsreihe Teile aufgepflanzt wurden, die nach ihren sichtbaren Eigenschaften dem neuen Wirte aus seinem regelrechten Leben nicht fremd waren und $d a B$ sich artfremde Teile um so besser hielten, je mehr ähnlichederneue Wirt schon regelrechterweise trug. Man kommt zu der Vermutung, daß sich gleiche Anteile des lebenden Stoffes auch bei artfremder Herkunft leichter anfeinander verpflanzen als ungleiche. Mengen- und Ortsänderung assimilatorischer Endgruppen eines Lebewesens erscheint leichter als ihre Qualitätsänderung.

Nicht nur das Leben des Finzelwesens, sondern auch das stammesgeschichtliche Leben der Art kann die Möglichkeit des Zusammenlebens bestimmter Teile schon bewiesen haben.

So gilt das, was wir hier für Wechselkröte-Erdkröte, Moorfrosch-Grasfrosch, Wasserfrosch-Grasfrosch fanden, auch für meine früheren Erfolge Girlitz-Kanarie, Grünling-Kanarie, SperlingKanarie, Lachtaube-Haustaube, Kaninchen-Hase, für Geschlechter und Haustierabarten.

Hat nicht die Stammesgeschichte für einen gewissen Stammesanteil die Fähigkeit gezeigt, beide Geschlechter zu tragen? 
Zeigen nicht die Stammformen der Haustiere in manchen Fällen die Haustierabarten schon in sich eingekapselt?

Bei einem wilden Kaninchen ist die Spitze der Haare schwarz. Das ist die schwarze Rasse. Schneidet man die Spitzen langsam zum Grunde vordringend $a b$, so erbält man die Bilder der braunen, gelben, blanen, weißen Rassen nacheinander als Schurergebnis.

Recht wunderbar findet man oft eine Abart in der anderen versteckt: schere ich etwa einem Schwarzlohkaninchen den obersten Teil seines weißgelblichen Bauchhaares, so tauchen die schwarzen Scheckenflecken des Scheckenkaninchens um seine Zitzen auf.

Wie verschiedenen Abarten gehörten die versteckten Eigenschaften an, die ich im Russenkaninchen entdeckte, als ich sein weißes Haar in farbiges verwandelte.

So verfolgt diese Arbeit den Weg der fruheren. Es scheint, als zeige sie für Arten einer Gattung zum Teile gleiche Verhältnisse, wie die fruberen Arbeiten sie für Geschlechter und Haustierformen und auch schon für nahestehende Arten wie Girlitz und Kanarienvogel zeigten.

Es zeigt sich ein Teil der Gründe, weshalb die Lebensstoffe dieser Formgruppen so wenig schädigend aufeinander wirken, sei es, daß ihre Sera sich schwach präzipitieren, sei es, daß ihre Gewebe sich verbältnismäßig gut aufeinander verpflanzen, sei es, daß sie sich kreuzen, daB ihre Bastarde mendeln, daß ihre Bastarde keine oder geringe Keimdrusenschädigung, Bastardunfruchtbarkeit zeigen, sei es schließlich, daß sie stammesgeschichtlich leicht auseinander entstehen.

\section{Die Verpflanzung als somatische Insertion in Beziehung zur blastogenen Insertion Rouxs. Die Bausteinaufnahme stammesgeschichtlich neuer Lebensstoffe.}

Es ist nicht zu bestreiten, daß die Verpflanzung als somatische Insertion in einem, wenn auch beschränkten Maße Aufklärung über die blastogene Insertion, eine Vorbedingung der erblichen Abänderung der Lebewesen und tiberhaupt tiber alle, insbesondere stammesgeschichtlich neuartige Bausteinaufnahme des Lebensstoffes gibt.

Die Bausteinaufnahme neuer Lebensstoffe meint Bausteine, die unmittelbare Teile des lebenden Stoffes werden, und will wissen, welche ganz neuartigen, stammesgeschichtlich neuartigen Bausteinverbindungen möglich sind, sei das Neuartige die Menge, die Stellung oder die Beschaffenheit, Qualität der Bausteine. 
Die Bausteinaufnahme beim reinen Wachsen, wobei Vorbandenes vermehrt wird, der Wiederersatz von Verlorenem bei Ernährung und Regeneration, die Wiederherstellung von schon Dagewesenem bei der Entwicklung auf dem Wege der Vererbung stehen in engster Beziehung zu unserem Gebiete.

Die blastogene Insertion Rouxs, d. h: die Aufnahme keimbildender Bausteine, ist mein Ausgangspunkt. Die Aufnahme artbildender Bausteine ist das letzte Ziel. Doch brauchen nach dem Sprachgebranch nicht alle stammesgeschichtlich nenen Lebensstoffe Keime und nicht alle Keime artbildend zu sein.

Wenn wir nun fragen: Welche Bausteine können sich mit einem Lebensstoffe (Plasma) neuartig verbinden, ohne das Leben zu vernichten? so muissen wir mit dem Einfachsten beginnen, wir müssen umgekehrt verfahren wie die Schutzstofforschung. Während diese meist mit Unterschieden arbeitet, welche die größten Schädigungen bedingen, müssen wir die Untersehiede suchen, welche die geringsten Schädigungen auf das Leben tiben, und diejenigen, welche überhaupt nicht mehr das Leben schädigen.

Hierftir können wir nun aus aller Artforschung, Entwicklungsmechanik, Ernährungsphysiologie und vielen anderem Schltisse ziehen.

Vielleicht der unmittelbarste Weg, die Bausteinaufnahme zu erforschen, ist die Mendelsche Vererbungsforschung mit ihrer Darstellung der Erbeinheiten.

Weitere Schlüsse muß die Schatzstofforschang zulassen, sei es die Darstellung der arteigenen Lösungsstoffe (Lysine), Niederschlaggstoffe (Präzipitine), Gegengifte (Toxine), der Überempfindlichkeit (Anaphylaxie) oder der Abwehrfermente.

Schon wiederholt habe ich aber auch daranf hingewiesen, dab gerade die Verpflanzungsforchung das Wirken der verschiedenen Lebensbausteine aufeinander zeigt.

Sie zeigt, wie verschiedene Arten lebenden Stoffes in echter Wachstumsverbindung aufeinander wirken und muß anch Schlisse gestatten, wie einzelne Bausteine, die sie einem bestimmten Lebensgefüge zufuhrt, sich diesem einfügen, welche Arten von Bausteinänderung sich schadlos einfügen, and welche nicht haften oder gar die Hauptanlage zerstören.

In dieser Richtung würden sich die Versuche dieser Arbeit und ihre Deutungen auch meiner früheren Arbeiten fördernd anschließen, falls die Regelmäßigkeit der Versuchsergebnisse sich weiterhin bestätigen sollte. 


\section{Zusammenfassung der Ergebnisse.}

1) Gleichlauf von Verpflanzung und Kreazung bei Rana temporaria L. und Rana arvalis Nilson, bei Bufo vulgaris Laur. und Bufo viridis Laur., eine neue Parallele zu meinen früheren bei Finken, Tauben, Huhnern, Hasenartigen.

2) Gleichlaúf bei in der Natur nicht kreuzbaren, aber innerhalb der Kreuzbarkeitsgrenzen stehenden Arten: Rana temporia L. und Rana esculenta L.

3) Verpflanzung als Einzelwesenreaktion bei Froschlurchen. Zweifel an dem Leben der nicht blutsverwandten, nicht körpereigenen, freien Hautverpflanzungen bei Lurchen.

4) Übereinstimmung der Verpflanzungs- und Präzipitinreaktion zwischen Maus und Ratte, Nichtübereinstimmung mit einer Kreuzungsangabe (Uhlenhuth, Walther Schultz, Iwanoff).

5) Weil der Gleichlauf nur bei gleichen Nebenbedingungen jeder Reihe neben der arteigenen Zusammensetzung des Plasmas sich darstellt, ist nur ein durchschnittlicher Gleichlauf von Kreuzung und System, Verpflanzung und System, Kreuzung and Verpflanzung darstellbar.

6) Anleitung, die Nebenumstände umzurechnen.

7) Farberscheinungen an freien Hautverpflanzungen der Froschlurche.

8) Schltisse von der Verpflanzung auf die Zusammenfugbarkeit der Plasmabestandteile, die blastogene Insertion Rouxs, die Artnenbildung.

\section{Nachtrag.}

Die Verpflanzungen Erdkröte auf Wechselkröte vom 21. X. 1915 und Grasfrosch auf Wasserfrosch vom 20. IX. 1915 wurden bis 5. VII. 1916, meinem Abmarsch ins Feld, erhalten.

\section{Erklärung der Abbildungen. \\ Tafel XII. \\ Rü ckenhautrerpflanzungen:}

Fig. 1. Von Moorfrosch (2) auf Grasfrosch (Vers. 12) am 75. Tage.

Fig. 2. Von Grasfrosch (1) anf Moorfrosch (Vers. 13) am 75. Tage.

Fig. 3. Von Erdkröte auf Wechselkröte (Vers. 19) am 75. Tage.

Fig. 4. Von Grasfrosch auf Erdkröte am 20. Tage (Vers. 23).

Fig. 5. Von Erdkröte anf Grasfrosch am 25. Tage (Vers. 24). 